\title{
The Effectiveness of Autologous Whole Blood Injection in the Treatment of Tendinopathy
}

\author{
Harpal Singh Uppal ${ }^{1 *}$, Adam Rumian ${ }^{1}$, Angelos Assiotis ${ }^{1}$, Simran Sahota $^{1}$, Ramandeep Singh \\ Saini $^{1}$ and Benjamin Dean ${ }^{2}$ \\ ${ }^{1}$ Lister Upper Limb Unit, Lister Hospital, United Kingdom \\ ${ }^{2}$ Nuffield Orthopaedic Centre, Oxford, United Kingdom \\ *Corresponding author: Harpal Singh Uppal, Lister Upper Limb Unit, Lister Hospital, Stevenage, Hertfordshire, United \\ Kingdom
}

\begin{tabular}{l}
\hline ARTICLE INFO \\
\hline Received: 慧 March 13, 2020 \\
Published: 彗 March 26, 2020 \\
\hline
\end{tabular}

Citation: Harpal Singh U, Adam R, Angelos A, Simran S, Ramandeep Singh S, et al., The Effectiveness of Autologous Whole Blood Injection in the Treatment of Tendinopathy. Biomed J Sci \& Tech Res 26(4)-2020. BJSTR. MS.ID.004399.

\section{Abstract}

\section{Aim:}

To assess the effectiveness of autologous whole blood injections in the treatment of tendinopathies in general.

\section{Objectives}

a) A comprehensive search to identify randomized controlled trials and case control studies on the subject of autologous whole blood injections (ABI) for tendinopathy. For example subacromial impingement, rotator cuff tendinopathy, tennis elbow, golfers elbow, patella tendonitis, Achilles tendonitis and plantar fasciitis.

b) Critical appraisal of above studies via validated assessment tools.

c) Synthesis of information from critical appraisal to generate more generalisable conclusions and implications for future practise.

Research Question: Are autologous whole blood injections effective compared to existing treatments in the management of tendinopathy?

Patients: Adults with tendinopathy. We excluded studies linking this with the use of a surgical procedure and studies using platelet rich plasma only, as the focus of this review is on whole blood products.

Intervention: The injection of autologous whole blood into an area of tendinopathy

Control: This included any comparative group. For example, corticosteroid injection, Non-steroidal anti-inflammatory injection, local anaesthetic and platelet rich plasma injection (PRP), Extracorporeal shockwave therapy.

Outcomes: Validated patient reported outcome measures (PROMS) as specific as possible to the particular type of tendinopathy being investigated.

\section{Background}

Tendinopathy is a common disorder with a reported prevalence of between 4 and $26 \%$ [1] affecting a wide variety of tendons and is characterized clinically with chronic pain, weakness and sometimes rupture. These disorders of tendon "comprise 30\% of all musculoskeletal consultations with a general practitioner" [2]. Despite the widespread morbidity from this condition the aetiology behind tendinopathy is still not entirely clear [3]. The disease process is thought to be caused by a "multifactorial process" [4]. Some of these factors may include non-physiological joint "mechanics re- 
sulting in recurrent microtrauma leading to tendon degeneration, micro tears and eventual failure of healing processes" [4]. These changes common to tendinopathies in general have been identified histologically with evidence of "fibroblast proliferation, vascular hyperplasia and angio fibroblastic hyperplasia" [4] without the presence of a chronic inflammatory component. The molecular pathology of tendinopathy is also characteristic with much work having been conducted over the last fifteen years on the role of matrix metalloproteinases [MMP] and their effect on proteoglycans [2]. That there is in vitro evidence that fluoroquinolone antibiotics are thought to be able to induce tendinopathies via their effect on MMP expression by tenocytes highlights the importance of biologically active mediators in tendinopathy [2].

Common sites for tendinopathy include the "rotator cuff, biceps tendon, the lateral and medial distal humeral epicondyle, patella tendon, Achilles tendon and the plantar fascia" [4]. The above clinical and histological findings are common to these conditions, indicating a possible common aetiology which goes someway to validate the similar forms of treatment that are commonly applied to these various conditions. Current treatments include "rest, anti-inflammatory medication, analgesia, orthotics, physiotherapy, local corticosteroid injection, extra-corporeal shockwave therapy and surgical debridement" [4]. There is no clear consensus on the optimal management of these tendinopathies. More recent treatments have focused upon the delivery of local growth factors to the site of tendinopathy [3]. "The basic science behind tendon healing following injury and the role of growth factors in modulating and initiating this process is well established" [4]. There are "three stages to tendon repair and regeneration" and all of these stages are influenced by local growth factors [2]. After an initial acute inflammatory response following tendon injury, migration of blood cells occurs and release of pro-inflammatory cytokines and angiogenic factors $[2,4]$. The proliferative stage continues with recruitment and proliferation of fibroblasts [2,4]. Fibroblasts are responsible for synthesis of collagen and proteoglycans essential for tendon repair. In the final remodeling stage the ratio of type one to type three collagen increases along with reorganization of collagen fibers into longitudinal bundles $[2,4]$. This final stage is usually complete at approximately six weeks post injury. A large number of local cytokines regulate these processes. These include transforming growth factor $\mathrm{B}(\mathrm{TGF}-\mathrm{B} 1)$, Basic fibroblast growth factor (b-FGF), Platelet derived growth factor [PDGF], Insulin like growth factor 1 (IGF-1),Vascular endothelial growth factor [VEGF], Epidermal growth factor [EGF] and Bone morphogenic proteins 12, 13 and 14 (BMP-12, BMP-13, BMP-14) $[2,4]$. These growth factors are found throughout the body but appear to be found in high concentrations within blood [4]. Much recent research has focussed on platelets in particular which are a rich and readily available source of PDGF, VEGF, EGF and BMP 12-14[2,4]. It must be noted that other factors not partic- ularly concentrated in platelets like TGF-B1, b-FGF and IGF-1 are also found within other components of blood [4]. There are a wide variety of methods via which these growth factors can be harvested and delivered to the zone of healing. Techniques that have received the most attention include leucocyte depleted low yield PRP, leucocyte rich high yield "buffy coat" PRP and ABI [5]. Every preparation is subtly different, and it must be appreciated that each of these preparations "contain many thousands of biologically active substances which have received very little attention in the literature concerning growth factor augmented soft tissue healing" [5].

Platelet rich plasma preparations are created following centrifugation of anti-coagulated autologous blood. The supernatant is discarded, and the platelet rich plasma is often activated for example with calcium chloride prior to its injection into the site of tendinopathy [5]. There are a number of features that have driven the use of platelet rich plasma. The cost of the treatment is low compared to the cost of recombinant growth factors [e.g. OP1 etc.]. Also, there are few barriers to its implementation in the form of governmental regulation as the injected product is autologous. In contrast to PRP, ABI involves the direct injection of whole blood with no preparation into the site of tendinopathy [5]. Though some of the previously mentioned growth factors are present in higher quantities in PRP than in ABI there is growing evidence that "less maybe more" [5], inferring that the optimal concentration of growth factors may in fact be present in whole blood as compared to PRP. The aim of this work is to systematically review the literature concerning $\mathrm{ABI}$ in the treatment of tendinopathy and if possible, to determine if the results of the trials identified can be synthesised into a more generalisable argument.

\section{Methods}

\section{Inclusion Criteria}

Randomized controlled trials and prospective studies with a comparator arm in English will be found that investigate.

\section{Population}

Human, Adults with tendinopathy affecting the rotator cuff, biceps tendon, medial epicondyle [Golfer's Elbow], Lateral epicondyle [Tennis elbow], Patella tendonitis, Achiles tendonitis or plantar fasciitis.

Intervention: One of the study interventions should involve the use of $\mathrm{ABI}$

\section{Control}

Any existing accepted treatments: Rest, Orthotics, Corticosteroid injection, Non-steroidal anti-inflammatory medication, Physiotherapy, Extra corporeal shockwave therapy and surgical debridement. 


\section{Outcome}

Validated PROMS specific to the joints affected by the tendinopathy being investigated. E.g. Constant score/Oxford Shoulder score for rotator cuff tendinopathy.

\section{Exclusion Criteria}

a) Animal studies, Studies not in English, Review articles, population groups with diseases other than tendinopathy, case series articles

b) Medline databases were interrogated via Pubmed from 1969 till the 16th of July 2019. MeSH headings for tendinopathy are a relatively recent entry into medline and as such not all references pertaining to tendinopathy are listed under the MeSH heading of tendinopathy. A combination of search terms was thus generated as follows and the above inclusion/exclusion criteria applied: autologous blood and ["Tennis Elbow" [Mesh] or "Fasciitis, Plantar"[Mesh] or "Shoulder Impingement Syndrome"[Mesh] or "Tendinopathy" [Mesh]]

c) Limits - Human and English studies only

The Consort statement will be used to critically appraise the selected papers and to help guide the process of synthesising this data into a more generalisable format.

\section{Critical Appraisal}

The two studies investigating plantar fasciitis were chosen for review [6,7]. By focusing the critical appraisal on plantar fasciitis this assignment can comprehensively review the subject of autologous blood injections to treat plantar fasciitis. Lee et al "Intralesional autologous blood injection compared to corticosteroid injection for treatment of chronic plantar fasciitis. A prospective, randomized, controlled trial" [7]. This prospective randomised controlled study from Kuala Lumpur compared the efficacy of autologous blood injections versus corticosteroid injection for the treatment of plantar fasciitis. The abstract provided a well laid out and organised summary of the trial design, methods, results and conclusions. The introduction explained the difficulties in treating plantar fasciitis and also provided information on the basic science behind the use of autologous growth factors to treat tendinopathy. This prospective study recruited 64 patients over a one-year period - three patients were lost to follow up with no explanation of why or of what efforts were made to follow these patients up. Inclusion and exclusion criteria were defined and applied.

The primary outcome measure was a form of visual analougue score [VAS]. VAS are a well validated form of assessing pain - the exact nature of the form used was not discussed and there is no evidence to show that the methods used to obtain a VAS were valid to the extent that these results be generalizable to other patients.
Other outcome measures involved a measurement of the pressure required to generate pain. This outcome measure has had no validation performed for it. One wonders why other outcomes scores such as the SF36, Foot and ankle ability measure [FAAM] or American Orthopaedic Foot \& Ankle Society foot and ankle score [AOFAS] were not used. Though outcome scores for foot and ankle pathology are not particularly well validated [8] it is perhaps more appropriate to use a moderately well validated outcome measure than a completely unvalidated measure. No sample size calculations appear to have been made. Randomization was performed via a computer-generated sequence, but no further detail has been provided concerning the nature of the sequence (e.g. if block randomization methods were used) and no information on how the sequence was used on actual patients. The patients and doctors providing treatment were not blinded but the outcome assessors were. Each group was assessed on an intention to treat basis. A table of demographics was provided along with inference statistics showing no obvious confounders in terms of the demographic data that was collected. Inference statistics were appropriately applied to the two outcome measures. The results showed a statistically significant difference in VAS and TT for both treatments. The $p$ value for this was significant. At six weeks and three months there appears to be a difference between the VAS and TT scores, with corticosteroid providing more pain relief. This difference is not present at 6 months. A confounder was introduced into the study as some patients had a second injection at three months. The criterion for this has not been explained and second injections have been given in a nonsystematic manner introducing bias into the results. In terms of patient harm, it was recognized that fat pad atrophy can be a complication of steroid treatment though this complication was not observed in the trial population. The trial was not registered with a trial registry, no funding sources were declared, and the full trial protocol does not appear to be available to the public.

The overall generalizability of this study is low primarily due to the poor choice of outcome measures. There are a variety of specific outcome measures available that should have been used to provide greater external validity. The study does provide some weight to the argument that steroid is more effective in the short term, however the similar outcomes at six months and the absence of longer term outcomes are consistent with research in other areas which demonstrates that steroid is not more effective in the medium term. Kalaci, et al. "Treatment of plantar fasciitis using four different local injection modalities: A randomized prospective clinical trial” [6].

In 2009 Kalaci reported results of a trial from Antalya in Turkey. The title and abstract fulfilled the consort criteria in providing a structured summary of the design, methods and results of this 'prospective randomized controlled' trial. The introduction explains the rationale for starting this trial in a clear and concise manner similar 
to the previous paper appraised and specific objectives with regard to aiming to demonstrate a difference in visual analogue scores as a primary outcome measure were declared. The patient group and interventions were well defined and had sufficient clarity to allow one to reproduce the study. Visual analogue pain scores were the primary outcome measure and there was no power calculation. One hundred patients were enrolled in this study. The first 25 consecutive patients underwent $\mathrm{ABI}$, the next consecutive 25 underwent local anesthetic injection with 'pepper potting' of the affected plantar fascia (this is a process of passing a needle multiple times through an area of tendinopathy), the third batch of 25 patients had steroid injection and the last group had steroid injection plus 'pepper potting. Clearly there has been no randomization whatsoever in this trial and it is prospective cohort study. The patients and the assessors were blind but obviously the doctors providing the treatment were not.

Surprisingly there was no loss to follow up. There was no flow chart to show patients progress through the trial and some baseline characteristics were displayed in tabular format. The results showed that all four treatments showed an improvement in pain scores. The two steroid treatment groups had less of a positive effect than the local anesthetic and autologous blood injection groups. P values are quoted as being significant but there is no description of which statistical tests were used which makes it hard to interpret the meaning of such results. There was also an attempt at creating a further two groups by pepper potting with $\mathrm{ABI}$ and with saline. These two patient groups found the injections too painful and were thus abandoned. Given that one of the included groups included saline injection with pepper potting I was very surprised to see that the trial had continued. If injection of saline was deemed too painful to allow the study to progress, then surely the injection of steroid in exactly the same way should also have been too painful. Clearly there were insufficient processes in place to assure patient safety during the course of this trial. This prospective randomized controlled trial was not randomized and given the $100 \%$ follow up and issues with patient safety in terms of spotting untoward complications it appears likely that the trial was not prospective either. The lack of power calculation, small numbers in each group, complete absence of explanation of which statistical tests were used when mean that this trial has little to no internal validity let alone external validity.

\section{Result and Discussion}

\section{Search Summary}

Twenty-six publications were identified with the above search strategy. Once review articles and studies pertaining solely to the use of platelet rich plasma or injection of cell culture suspensions [as opposed to autologous blood injections] were excluded five studies were identified. The flow chart below describes the appli- cation of the exclusion criteria.

\section{Studies Identified}

a) 10 review articles excluded

b) One article on injection of cultured tenocytes excluded [9]

c) 6 studies investigating platelet rich plasma excluded

d) One study on cerebral palsy patients excluded [10]

e) Four studies were not randomized and had no control group i.e. they were case series [11-14]

\section{Studies Left for Review}

All studies were randomized controlled trials, 3 studies investigated lateral epicondylitis $[5,15,16]$ and 2 investigated plantar fasciitis [6,7].

\section{Summary of Evidence}

With regard to lateral epicondylitis there are three randomized controlled trials. In 2011 Creaney [5] published a trial comparing the patient derived tennis elbow evaluation score in patients who received autologous blood injections or platelet rich plasma injections to treat chronic lateral epicondylitis that had failed conservative management. This study showed that both treatments provided a significant improvement in function post injection. The patient related tennis elbow evaluation score was the primary outcome measure. Platelet rich plasma injection showed an improvement of 33 points (95\% confidence interval 28.2 to 37.8 ) whilst ABI showed an improvement of 37.7 points (95\% confidence interval 32.2 to 43.3). The autologous blood injection group had superior function, but this was not statistically significant.

In 2010 Kazemi [15] published results of a randomized controlled trial from a teaching hospital in Tehran. This study compared autologous blood injection to steroid injection in new patient referrals with tennis elbow. The outcome measures involved were VAS scores, the quick DASH score, the Nirschl score and muscle strength testing. The results of this trial show that both treatments work very well to relieve pain but also surprisingly show that autologous blood injections were in this patient group overwhelmingly superior to steroid injection for all outcome measures. All P values when comparing the two groups were $<0.001$. Given the small sample sizes involved finding such overwhelmingly positive results in favour of autologous blood injections, which are at odds with other studies on similar patients, appear to be perhaps slightly unbelievable!

Ozturan [16] reported findings of a study comparing ABI to steroid injection to extracorporeal shock wave therapy in the treatment of tennis elbow. Sixty patients were randomized (with no description of randomization and no power calculation) to the three 
treatments. The steroid injection technique involved perforating the area of tendinopathy at least five times. Dry needling in itself is a treatment for tendinopathy [6] and this is a significant confounder. The trial showed that all three studies showed an improvement in visual analogue scores post provocative Thompson testing-a non-validated measure of elbow function. Steroid injection was superior to the other two treatments at 4 weeks $[p<0.01]$ these differences disappeared at 12 weeks and by 26 weeks the ABI group and shockwave group showed reduced pain compared to steroid injection $[p<0.01]$ - this difference was maintained until 52 weeks the end point of the study. The study recommends the use of autologous blood injections on the grounds that it is superior to steroid injection and equivalent in efficacy to shockwave therapy with the benefit of being cheaper than shockwave therapy.

\section{Implications for Clinical Practise}

To date there are five randomized studies comparing autologous blood injection with existing treatments for tendinopathy. None of these studies have shown adequate demonstration of power calculation, randomization or blinding. Most of these studies fail to account for obvious confounding variables such as the effect of dry needling in the process of injection. The evidence base seems to suggest that most of the current treatments available are effective in the treatment of tendinopathy. Four out of five studies suggest that $\mathrm{ABI}$ is superior or equivalent to control groups. Given the small sample sizes and large number of confounders there is significant scope for both type 1 and type 2 error in conclusions drawn from the current evidence base. Compared to other treatments ABI is remarkable in its low price and ease of application - a point which is raised repeatedly $[4,6,16]$. From this small and fairly weak evidence base one can conclude that ABI is likely to be similar in its acceptability to patients compared to controls [15] an assertion which also passes the test of face validity. A significant problem is the use of the steroid comparator as high-quality research has shown that although steroid is superior to placebo in the short term, it is harmful in the medium and longer term [Coombes JAMA]

\section{Implications for Future Research}

A key failing in all of these studies is the lack of a power calculation and poor descriptions of randomization. Unfortunately, we would not recommend using any of the currently described datasets as the base for a power calculation for further studies due to the paucity of validated outcome measures used in the studies described above. A pilot study is required assessing a type of tendinopathy which has a well validated outcome measure associated with it. Given its high incidence within the population [1] and the development of very well validated outcome measures such as the Oxford shoulder score [17] and the Constant score [17] rotator cuff tendinopathy would be the best starting point for this investigation into tendinopathy. This pilot study should compare ABI with an adequate comparator. A significant failure of the summarized studies is the adequacy of the comparator arms; ultimately a placebo comparator would be optimal in order to assess the effectiveness of $A B I$ and this could be done alongside a steroid injection arm, as this is the most widely used in current clinical practice. [1]. The results of this pilot study should guide the power calculation in the design of a randomized controlled trial to compare the effect of ABI vs. placebo vs steroid injection in the treatment of rotator cuff tendinopathy. This study would definitively show if $\mathrm{ABI}$ is effective in the treatment of tendinopathy and if it is superior to existing treatment.

\section{Competing Interests}

The authors declare that they have no competing interests.

\section{Acknowledgement}

Not applicable.

\section{References}

1. Murphy RJ, Carr AJ (2010) Shoulder pain. Clin Evid.

2. Riley G (2008) Tendinopathy from basic science to treatment. Nat Clin Pract Rheumatol 4(2): 82-89.

3. Rabago D, Best TM, Zgierska AE, Zeisig E, Ryan M, et al. (2009) A systematic review of four injection therapies for lateral epicondylosis: prolotherapy, polidocanol, whole blood and platelet-rich plasma. Br J Sports Med 43(7): 471-481.

4. Kampa RJ, Connell DA (2010) Treatment of tendinopathy: is there a role for autologous whole blood and platelet rich plasma injection? Int J Clin Pract 64(13): 1813-1823.

5. Creaney L, Wallace A, Curtis M, Connell D (2011) Growth factor-based therapies provide additional benefit beyond physical therapy in resistant elbow tendinopathy: A prospective, single-blind, randomised trial of autologous blood injections versus platelet-rich plasma injections. Br J Sports Med.

6. Kalaci A, Cakici H, Hapa O, Yanat AN, Dogramaci Y, et al. (2009) Treatment of plantar fasciitis using four different local injection modalities: a randomized prospective clinical trial. J Am Podiatr Med Assoc 99(2): 108-113.

7. Lee TG, Ahmad TS (2007) Intralesional autologous blood injection compared to corticosteroid injection for treatment of chronic plantar fasciitis. A prospective, randomized, controlled trial. Foot Ankle Int 28(9): 984-990.

8. Goldstein CL, Schemitsch E, Bhandari M, Mathew G, Petrisor BA (2010) Comparison of different outcome instruments following foot and ankle trauma. Foot Ankle Int 31(12): 1075-1080.

9. Clarke AW, Alyas F, Morris T, Robertson CJ, Bell J, et al. (2011) Skinderived tenocyte-like cells for the treatment of patellar tendinopathy. Am J Sports Med 39(3): 614-623.

10.Logan LR, Klamar K, Leon J, Fedoriw W (2006) Autologous blood injection and botulinum toxin for resistant plantar fasciitis accompanied by spasticity. Am J Phys Med Rehabil 85(8): 699-703.

11. James SL, Ali K, Pocock C, Robertson C, Walter J, et al. (2007) Ultrasound guided dry needling and autologous blood injection for patellar tendinosis. Br J Sports Med 41(8): 518-521; discussion 22. 
12. Suresh SP, Ali KE, Jones H, Connell DA (2006) Medial epicondylitis: Is ultrasound guided autologous blood injection an effective treatment? $\mathrm{Br}$ J Sports Med 40(11): 935-939; discussion 9.

13. Connell DA, Ali KE, Ahmad M, Lambert S, Corbett S, et al. (2006) Ultrasound-guided autologous blood injection for tennis elbow. Skeletal Radiol 35(6): 371-377.

14. Edwards SG, Calandruccio JH (2003) Autologous blood injections for refractory lateral epicondylitis. J Hand Surg Am 28(2): 272-278.

15. Kazemi M, Azma K, Tavana B, Rezaiee Moghaddam F, Panahi A (2010) Autologous blood versus corticosteroid local injection in the short-term

ISSN: 2574-1241

DOI: 10.26717/BJSTR.2020.26.004399

Harpal Singh Uppal. Biomed J Sci \& Tech Res

(C) This work is licensed under Creative

Submission Link: https://biomedres.us/submit-manuscript.php treatment of lateral elbow tendinopathy: A randomized clinical trial of efficacy. Am J Phys Med Rehabil 89(8): 660-667.

16. Ozturan KE, Yucel I, Cakici H, Guven M, Sungur I (2010) Autologous blood and corticosteroid injection and extracoporeal shock wave therapy in the treatment of lateral epicondylitis. Orthopedics 33(2): 84-91.

17. Harvie P, Pollard TC, Chennagiri RJ, Carr AJ (2005) The use of outcome scores in surgery of the shoulder. J Bone Joint Surg Br 87(2): 151-154.

\begin{tabular}{ll}
\hline RESEARCHES & Assets of Publishing with us \\
\hline BIOMEDICAL & Global archiving of articles \\
\hline & - Immediate, unrestricted online access \\
\hline
\end{tabular}

